\title{
The GLAST Background Model
}

\author{
J. F. Ormes ${ }^{1}$, W. Atwood ${ }^{2}$, T. Burnett ${ }^{3}$, E. Grove ${ }^{4}$, F. Longo ${ }^{5}$, J. McEnery ${ }^{6}$, T. \\ Mizuno $^{7} \&$ S. Ritz ${ }^{6}$ on behalf of the GLAST mission team
}

\author{
(1)University of Denver; (2)University of California at Santa Cruz; (3)University of Washington; \\ (4)Naval Research Laboratory; (5)Instituto Nazionale di Fisica Nucleare (INFN)- Pisa,; \\ (6)NASA Goddard Space Flight Center; (7)Hiroshima University
}

\begin{abstract}
In order to estimate the ability of the GLAST/LAT to reject unwanted background of charged particles, optimize the on-board processing, size the required telemetry and optimize the GLAST orbit, we developed a detailed model of the background particles that would affect the LAT. In addition to the well-known components of the cosmic radiation, we included splash and reentrant components of protons, electrons ( $\mathrm{e}^{+}$and e-) from $10 \mathrm{MeV}$ and beyond as well as the albedo gamma rays produced by cosmic ray interactions with the atmosphere. We made estimates of the irreducible background components produced by positrons and hadrons interacting in the multilayered micrometeorite shield and spacecraft surrounding the LAT and note that because the orbital debris has increased, the shielding required and hence the background are larger than were present in EGRET. Improvements to the model are currently being made to include the east-west effect.
\end{abstract}

Keywords: Cosmic ray, gamma ray.

PACS: 95.55.ka; 95.85.Pw; and 95.85.Ry

The details of the GLAST background flux model are outlined in this brief paper. The model was developed to estimate the trigger rate and downlink rates that will be produced and to optimize the orbital parameters. It is also useful for evaluating the irreducible background in the LAT. Protons $>100 \mathrm{MeV}$ and e+ $>20 \mathrm{MeV}$ can interact in the multilayered micrometeorite shield ( $2 \mathrm{x}$ as thick as that of EGRET) and make photons indistinguishable from celestial photons. Positrons can annihilate in flight; electrons and positrons can undergo a high fractional loss of energy by Bremsstrahlung; and protons can have interactions in which no resultant particle passes through a charge identifying counter (especially the anti-coincidence detector - ACD) while producing a gamma ray that is detected by the LAT. Also, in some orientations of the spacecraft, Earth Albedo gamma rays may not all be eliminated. The several components of this background are expected to vary along the orbit. The relative importance of each component will be determined by the orbit position and the arrival direction in local zenith pointing coordinates. By studying modulations of the background as a function of orbital parameters and arrival direction, the magnitude of these effects will be understood. The east-west effect will be helpful in separating components caused by particles of the opposite sign.

FIGURE 1. Legend: Total (open circles), GCR protons (filled triangle up), He+heavies (open diamond), GCR electrons (filled square), albedo protons (open triangle), albedo $\mathrm{e}^{+}$ (filled circle), albedo e- (open square), and albedo gammas (filled triangle down). Left Panel: Orbit averaged background $E^{*}$ flux for estimating trigger rates and required downlink bandwidth; Right panel: Input E*Flux plot for events that end
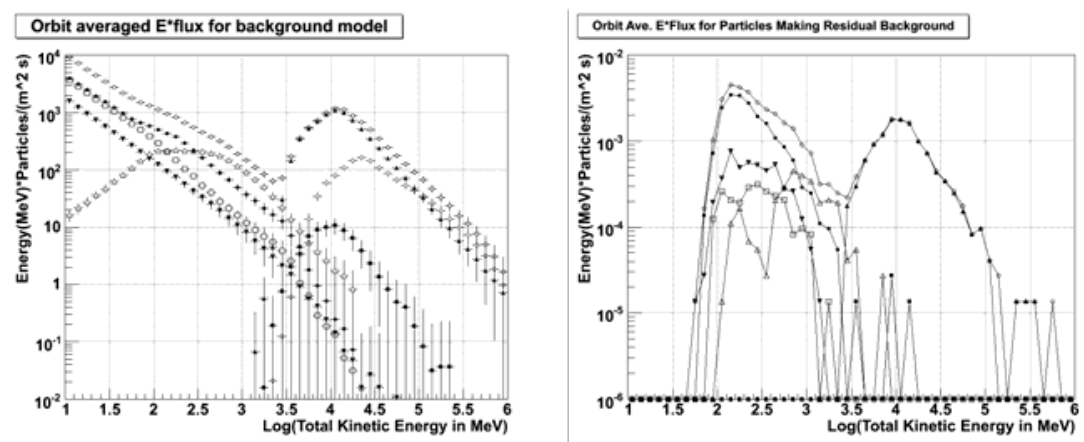

up as residual gamma ray background by particle type. Comparing the two plots, we see that GCR protons are reduced by the factor $2 \times 10^{-6}$ and one in a little over 100,000 positrons are creating a residual background. 
We model all components of galactic cosmic rays including electrons, positrons, anti-protons and nuclei up to iron as well as Earth albedo spectra for splash and reentrant electrons, positrons and protons in geomagnetic latitude bins of 0.1 radians. The model is expanded from one used for simulating backgrounds in a balloon borne test of a GLAST module (5). The galactic cosmic ray and albedo spectra above $150 \mathrm{MeV}$ are based on measurements by AMS (1) and, below $150 \mathrm{MeV}$, on a series of Russian experiments (3, 4, 7, and 8). Reentrant and splash albedo are modeled separately for e-, e+ and protons. Albedo positron spectra are shown as an example in Fig. 2 Left.
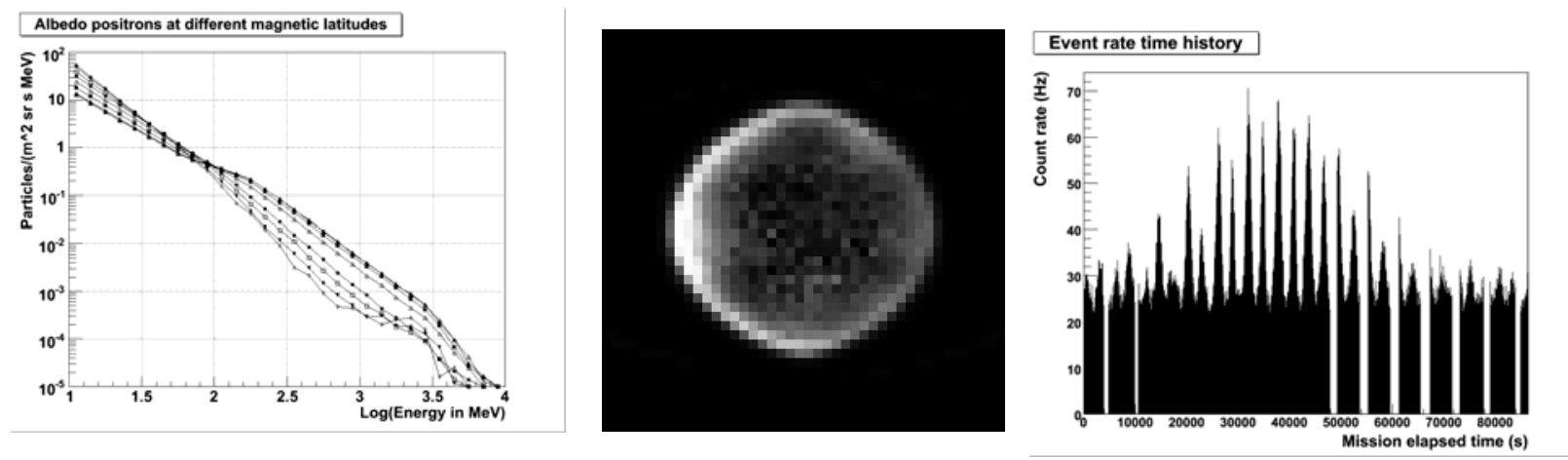

FIGURE 2. Left: Albedo positrons sorted by latitude. Legend: latitude in radians: 0.0 to 0.1 (filled triangle up), 0.1 to 0.2 (open triangle up), 0.2 to 0.3 (filled square), 0.3 to 0.4 (open triangle up), 0.4 to 0.5 (filled circle), 0.5 to 0.6 (open square), 0.6 to 0.7 filled triangle down), and 0.7 to 0.8 (open diamond). Center: Map of the albedo gamma rays from the interaction of cosmic rays with the Earth's atmosphere. Right: The modeled trigger rate as a function of time for one day.

The Earth albedo gamma-ray spectrum is seen in Fig. 2 Center image (6). The east-west effect is clearly seen in Fig. 2 Center image (6). We have incorporated the east-west effect for this background component. The east-west effect will be implemented soon for charged particles.

The model allows us to estimate the trigger rates under various conditions. Fig. 2 Right shows the total trigger rate as a function of time for a typical day. The rate drops to zero during SAA passages. The orbital period is $5400 \mathrm{~s}$. There are two peaks per orbit from the highest latitudes (north and south).

\section{CONCLUSIONS}

- The background model accurately reflects the measured galactic cosmic ray fluxes from $10 \mathrm{MeV}$ up to $100 \mathrm{GeV}$

- Earth albedo positrons and electrons are modeled down to $10 \mathrm{MeV}$, but the uncertainties are increasingly large as we go below $\sim 200 \mathrm{MeV}$ reaching $+/-50 \%$ at $10 \mathrm{MeV}$. The sensitivity to background falls rapidly below 100 $\mathrm{MeV}$.

- Estimating the uncertainty in the irreducible background depends on knowing the flux of positrons - results from Pamela will be able to improve the model and extend it to higher latitudes.

- The east-west effect is being implemented.

- This model is far more detailed than any other available model for satellites in low Earth orbit.

\section{REFERENCES}

1. Aguilar et al., 2002, Physics Reports 366, 331-405.

2. Bidoli et al.2002, Annales Geophysicae, 20, issue 10, 1693-1697.

3. Dmitriev, A.V., et al., 1998, Advances in Space Research, Vol.21, No.12, 1797-1800.

4. Mikhailov, 2002, J.of Mod.Phys.A, 17, Ns12-13, 1695-1704.

5. Mizuno, T. 2004, ApJ, 614, 1113-1123.

6. Petry, D., 2005, AIP Conf. Proc. 745, 709-714, astro-ph/0410487.

7. Voronov, S.A., et al., 1991, Cosmic Res., Engl. Transl., 29(4), 567-575.

8. Voronov, S.A., S.V.Koldashov, and V.V.Mikhailov, 1995, Cosmic Res., Engl.Transl., 33(3), 329-331. 